workers $(18 \%)$ more often than FSW $(0 \%, \mathrm{p}<0.01)$ and MSM $(3 \%$, $\mathrm{p}<0.01)$.

Conclusion MSW are at a high risk for contracting and transmitting STI from and to their clients, but also from and to non-commercial (female) contacts and within their own MSW network. This makes them a potential bridging population for STI transmission. While the MSW population is largely hidden, we prove that it is feasible to reach them with care.

\section{P3.114 ARE MALE SEX WORKERS IN TEL-AVIV, ISRAEL AT HIGHER RISK TO GETTING STI AND HIV THAN MEN WHO HAVE SEX WITH MEN}

doi:10.1136/sextrans-2013-051184.0573

'Z Mor, ${ }^{2,3} \mathrm{M}$ Dan. 'Ministry of Health, Ramla, Israel; ${ }^{2}$ Wolfson Medical Center, Holon, Israel; ${ }^{3} T e l$ Aviv University, Tel Aviv, Israel

Background The number of men who have sex with men (MSM) newly diagnosed with HIV in Israel has quadrupled since 2000. Male sex workers (MSW) are often associated with risk behaviours and STI/HIV infections; thus, they may play a role as core group transmitters. This study aimed to explore knowledge, attitudes and sexual-practises of MSW in Tel-Aviv in comparison with MSM, classified by their risk-behaviours, and to outline attributes related to STI/HIV-prevalence.

Methods MSW were recruited for this cross-sectional study from designated street venues and gay-dating internet-site. MSM were recruited from gay-related venues and divided into high-risk MSM (HRMSM) if they performed unprotected anal intercourse in the last six-months with a HIV-discordant/unknown partner, and to low-risk MSM (LRMSM). Each participant completed a questionnaire and was tested for STI/HIV.

Results Of 87 MSW and 635 MSM approached, 53 (60.9\%) and 235 (37.0\%) participated, respectively. Street-MSW (N = 32) had more female sex partners and were more drug-dependant than internetMSW ( $N=21)$. No differences were found in their knowledge regarding STI/HIV transmission, practises and STI/HIV burden.

Compared to HRMSM, MSW had different demographic attributes and demonstrated more realistic perception regarding the risk to getting STI/HIV, while no differences were found in their knowledge and sexual practises. STI and HIV burden among MSW, HRMSM $(\mathrm{N}=119)$ and LRMSM $(\mathrm{N}=116)$ were $28.3 \%, 23.5 \%$, $10.3 \%$, and $5.6 \%, 9.2 \%, 0 \%, p=0.009$ and 0.001 , respectively.

Multivariate models evaluating attributes associated with HIV/ STI-diagnosis did not find sex-work to be significant if the variable used was MSW vs. HRMSM, regardless of the adjustments performed. However, when MSW vs. LRMSM was used in the model, sex-work was associated with STI/HIV-diagnosis.

Conclusions Street-MSW and internet-MSW shared comparable sexual practises and no difference was found in their STI/HIV burden. Likewise, MSW and HRMSM had comparable sexual practises and no difference in STI/HIV prevalence.

\section{P3.115 HIV AND STI PREVALENCE AMONG COMMERCIAL SEX WORKERS}

doi:10.1136/sextrans-2013-051184.0574

A T Sosela. Sinza Youth Peer Educators, Dar-es-salaam, Tanzania

Background Although there is increasing evidence of the risks associated with HIV infection and transmission among CSW in Tanzania. nothing is known about other sexually transmitted infections (STIs) in this population.

Methods As a strategy to address high HIV prevalence rate among the CSWs, SIYOPE established a programme in Dar-es-salaam in 2009 to 2011. The project involved HIV prevention interventions in a coordinated manner integrating both clinical services and intensified community outreach programme targeting the CSWs. Some of the key steps undertaken by the project in developing an integrated health services for CSWs include community Mobilization \& CSWs, care and Support. Since its inception the project a total of 307 CSW were recruited between April and October Out of them, $96 \%$ have been screened for HIV, 94\% have been screened for STI, $55 \%$ have been screened for TB and 56 are accessing FP services. Further 75 CSWs have tested positive, 30 are on ARVs and the project has formed 2 support groups for women living with HIV, In addition the project continues to address local-level policy issues that hinder access to services among the CSWs as well as stigma and discrimination through campaigns and engagement of community police.

Results Most of the CSW were aged 14-25years and a large proportion (95\%) reported having multiple male partners with Whom they often had unprotected sex. 31\% reported STIs in the past 12 months, while 26 reported STI symptoms at the time of the outreach activity. Weighted prevalence of STIs ranged from $0.5-1.9 \%$ for syphilis, $4.2-8.9 \%$ for gonorrhoea, $0-34.5 \%$ for Chlamydia.

Conclusion There is a large unmet need for CSW in Tanzania. This calls for an urgent need for targeted screening and vaccination to prevent The untoward HIV and STIs among CSW in Tanzania.

\section{P3.116 RISK FACTORS FOR MYCOPLASMA GENITALIUM AMONG FEMALE SEX WORKERS IN NAIROBI, KENYA}

doi:10.1136/sextrans-2013-051184.0575

'A Alakija, ' $\mathrm{J}$ Ting, ${ }^{2} \mathrm{~N}$ Mugo, ${ }^{2} \mathrm{~J}$ Kwatampora, ${ }^{1} \mathrm{~F}$ S Behets, ${ }^{3} \mathrm{C}$ Hill, ${ }^{2 \mathrm{M}}$ Chitwa, ${ }^{4} \mathrm{~S}$ Patel, 'M Gokhale, ${ }^{2} \mathrm{~J}$ Kimani, ${ }^{1,5} \mathrm{~J} \mathrm{~S}$ Smith. 'Department of Epidemiology, Gillings School of Global Public Health, University of North Carolina at Chapel Hill, Chapel Hill, NC, United States; ${ }^{2}$ Kenyatta National Hospital/University of Nairobi, Nairobi, Kenya; ${ }^{3}$ Gen-Probe Incorporated, San Diego, NC, United States; ${ }^{4}$ Department of Obstetrics, Gynecology and Reproductive Sciences, University of California, San Francisco, CA, United States; ${ }^{5}$ Lineberger Comprehensive Cancer Center, Chapel Hill, NC, United States

Background Prevalence of Mycoplasma genitalium is higher in vulnerable populations of women in low-resource settings; predictors for infection, however, are not well understood. This study aimed to determine the prevalence of Mycoplasma genitalium and its associated risk factors among female sex workers in Nairobi, Kenya.

Methods A total of 350 FSW aged 18-50 years old participated in this cross-sectional study from 2009 to 2011. A questionnaire was administered at baseline to obtain information on sociodemographics and sexual behaviours. Physician-collected cervical samples were tested for $M$. genitalium mRNA and other sexually transmitted infections using transcription-mediated amplification-based APTIMA assays (Hologic/Gen-Probe Inc.). Cervical cytology was conducted using physician-collected specimens and classified according to the Bethesda criteria.

Results The median age in the study was 28 (range: 18-48). Prevalence of $M$. genitalium was $12.7 \%$. In the multivariate model, younger age was associated with a higher risk of $M$. genitalium, whereby women greater than 30 years old were less likely to have infection (OR [95\% confidence interval] $=0.3$ [0.1.0.9] versus women less than 30 years old). Women who reported using condoms at least "most of the time" were more likely to be infected with $M$. genitalium than women who reported using condoms "half the time or less." Relative to those with normal cytology, women with cytology results of either high-grade squamous intraepithelial lesions or squamous cell carcinoma had a higher prevalence of $M$. genitalium ( $23.1 \%$ vs. $16.2 \%$ ), although effect estimates were relatively imprecise.

Conclusions Younger women within a vulnerable population appear to be at greater risk of $M$. genitalium infection. The reverse association between condom use and $M$. genitalium may be due to 